\title{
The Static and Dynamic Mechanical Properties of Magnetorheological Silly Putty
}

\author{
Fei Guo, ${ }^{1}$ Cheng-bin Du, ${ }^{1}$ Guo-jun $\mathrm{Yu}^{2}{ }^{2}$ and Run-pu Li ${ }^{1}$ \\ ${ }^{1}$ Department of Engineering Mechanics, Hohai University, Nanjing 210098, China \\ ${ }^{2}$ Faculty of Civil Engineering and Mechanics, Jiangsu University, Zhenjiang 212013, China \\ Correspondence should be addressed to Cheng-bin Du; cbdu@hhu.edu.cn
}

Received 14 May 2016; Revised 22 June 2016; Accepted 26 June 2016

Academic Editor: Antônio G. de Lima

Copyright (c) 2016 Fei Guo et al. This is an open access article distributed under the Creative Commons Attribution License, which permits unrestricted use, distribution, and reproduction in any medium, provided the original work is properly cited.

\begin{abstract}
A novel magnetorheological material defined as magnetorheological Silly Putty (MRSP) is prepared by dispersing soft magnetic particles into Silly Putty matrix with shear stiffening property. Static mechanical properties including creep and stress relaxation and dynamic rheological properties of MRSPs are tested by rheometer. The experimental results indicate that the external magnetic field exerts significant influence on the creep and relaxation behaviors. Moreover, the storage modulus of MRSPs increases sharply in response to the external stimuli of increasing angular frequency automatically and can be enhanced by external magnetic field. Besides, temperature plays a key role in shear stiffening and magnetorheological effect of MRSPs. Furthermore, considering the obstruction to the particle chains formation induced by Silly Putty matrix, a nonperforative particle aggregated chains model is proposed. The model curve is in consistency with experimental data, which means it can describe magnetoinduced behavior of MRSPs well.
\end{abstract}

\section{Introduction}

Silly Putty is an intelligent material that exhibits obvious shear stiffening property. It can be easily kneaded, much like dough, into any shape desired. However, if a shock or impulsive load is applied to the putty, it will stiffen and even shatter. In rheological terms the experimenter is distorting the Silly Putty over a range of Deborah numbers. This nondimensional parameter describes the ratio of the fluid relaxation time scale to that of the experimental time scale. A high Deborah number therefore corresponds to a fast experiment in which the load or impulse is applied over a very short time scale. Due to its unique rheological property, Silly Putty is becoming a promising material for a special damper called shock transmission unit (STU) widely used in multispan bridge [1].

Shear stiffening property of Silly Putty has been investigated by many researchers. Cross [2] proposed a threeelement parameter model to describe the compression of Silly Putty at different compression speeds; Goertz et al. [3] developed a mechanical model for Silly Putty based on three Maxwell elements in parallel to study the influence of the temperature on the rheological property and found that the mechanical properties shifted significantly with temperature. Wang et al. $[4,5]$ prepared a novel shear stiffening gel (STG) with polydimethylsiloxane (PDMS), boric acid, benzoyl peroxide (BPO), and different additives such as calcium carbonate and carbon nanotube. The stiffening mechanism of STG was also explained in respect of chemical bond.

Magnetorheological materials are a class of smart materials that have usually been prepared by dispersing magnetic particles into different polymer matrices. The mechanical properties of magnetorheological materials can be controlled by an externally imposed magnetic field. Due to the controllable rheological property, magnetorheological materials have thus been widely applied in dampers, isolators, and different vibration controllers. However, most solid-state magnetorheological materials cannot present considerable tunability. Interestingly, on the basis of the fabricated STG, Wang et al. $[6,7]$ firstly developed a magnetically responsive shear stiffening gel with excellent shear stiffening performance and magnetorheological effect. This novel magnetically responsive shear stiffening gel could provide credible tunability with external stimuli. 
Besides, some theoretical models have also been developed to describe the magnetoinduced behavior of magnetorheological materials. Jolly et al. [8] proposed a dipole model based on the magnetic interactions between two adjacent particles. Shen et al. [9] presented a mathematical model to represent the stress-strain relationship of magnetorheological elastomer. The model considered all the dipole interactions in a chain and the nonlinear properties of the host composite. Chen et al. [10] developed a finite-column model to describe the relationships between the microstructures and the viscoelastic properties. However, for magnetorheological Silly Putty (MRSP) of soft magnetic particles dispersing in Silly Putty matrix, when external magnetic field strength increases, the internal structure changes unlike that of magnetorheological elastomers, and the chain structures are not pulling through like magnetorheological fluids.

In this work, several MRSP samples were prepared by dispersing carbonyl iron particles into Silly Putty matrix. Both static and dynamic mechanical properties were tested by rheometer. The static mechanical testing indicated that MRSPs exhibit obvious creep and stress relaxation behaviors and external magnetic field strength plays a key role in creep compliance and relaxation modulus. The dynamic mechanical testing revealed that MRSPs display both good shear stiffening property and high magnetorheological effect; furthermore, influence factors of mechanical properties for MRSPs including temperature and particle concentration were analyzed. Finally, a nonperforative particle aggregated chains model considering the obstruction to the particle chains formation induced by Silly Putty matrix was proposed to describe magnetoinduced behavior of MRSPs. Due to the tunable shear stiffening and magnetorheological effect, MRSPs are candidates for applications in dampers and STUs widely used in vibration control of buildings and bridges.

\section{Experimental Section}

2.1. Materials. Dow Corning 3179 Dilatant Compound purchased from Dow Corning Co., Ltd. was used as the Silly Putty matrix. The composition of the silicone material was as outlined in Table 1. The soft magnetic particles carbonyl iron (CI) of average size $3.5 \mu \mathrm{m}$ was purchased from Jiangsu Tianyi Ultra-Fine Metal Powder Co., Ltd., Xuyi, China.

2.2. Preparing and Testing the MRSPs. The dilatant compound as matrix and different volume fractions of CI particles as fillers were homogeneously mixed by a two-roll mill (Nantong Hailite Rubber Machinery Inc., China, model XK$160)$ at room temperature. For mechanical mixing method, up to six different volume fractions had been considered in this study: $0,6.98,10.11,15.84,20.80$, and $27.29 \%$ and the prepared samples were marked as MRSP-0, MRSP-1, MRSP-2, MRSP3, MRSP-4, and MRSP-5 in sequence. For particle filled materials like MRSPs, dispersion quality is crucial in the rheological properties [11]. Therefore, in this work, Hitachi S4800 scanning electron microscope (SEM) was used to observe the internal microstructure of MRSPs. MRSP sample and the SEM image are displayed in Figure 1. It can be obtained that CI particles are uniformly dispersed in Silly Putty matrix
TABLe 1: The composition of Silly Putty matrix.

\begin{tabular}{lc}
\hline Composition & Weight percentage \\
\hline PDMS & $65 \%$ \\
Silica & $17 \%$ \\
Thixotrol & $9 \%$ \\
Boric acid & $4 \%$ \\
Glycerine & $1 \%$ \\
Titanium dioxide & $1 \%$ \\
Dimethyl cyclosiloxane & $1 \%$ \\
\hline
\end{tabular}

approximately and MRSP sample presents isotropic feature due to great mechanical force provided by milling roller XK160.

Static mechanical properties including creep and stress relaxation and dynamic rheological properties were carried out using a commercial rheometer (Physica MCR 302, Anton Paar Co., Austria). During the testing procedure, a parallel plate PP20 with a diameter of about $20 \mathrm{~mm}$ was used and the gap of $1 \mathrm{~mm}$ was kept all the time. At the same time, a controllable magnetic field was generated by an external coil. Besides, all the testing samples were kept at approximately the same volume value. Most of all, as a kind of rate-dependent material, MRSPs exhibit obvious transition from polymeric fluids on the condition of static state or low shear rate to solidlike polymers under high shear rate. Furthermore, the applied shear strain was very small, especially for dynamic testing, the shear strain was only $0.1 \%$. So, no matter for polymeric fluid state or solid-like state of MRSPs, the fracture or wall-slip effects were not considered in this work.

\section{Results and Discussion}

3.1. Creep and Relaxation Behavior of MRSPs. Figures 2(a) and 2(b) show the creep characteristic of MRSP-4 on the condition of different initial stress and zero magnetic field at room temperature. Creep phase (0-300 s) and recovery phase (300-900 s) are explicitly demonstrated in Figure 2(a). MRSP presents more obvious deformation (maximum strain $20.6 \%$ of $300 \mathrm{~Pa}$ ) but lower creep compliance with higher initial stress as shown in Figure 2(b). In the creep phase, creep compliance $J(t)$ of MRSP increases with time gradually.

Figures 3(a) and 3(b) show the effect of different external magnetic field strength on creep characteristic of MRSP-4 with the initial stress $300 \mathrm{~Pa}$. It proves that external magnetic field strength can substantially confine creep deformation and creep compliance of MRSP. With an external magnetic field of $23.9 \mathrm{kA} / \mathrm{m}$, the maximum strain sharply decreases to only $0.375 \%$ comparing with the value $20.6 \%$ of zero magnetic field. That is because CI particles arrange in chain structure with external magnetic field, thus inducing the generation of magnetoinduced modulus which contributes to the reciprocal of creep compliance $J(t)$.

Relaxation modulus of MRSP-4 as a function of time under different initial strain and external magnetic field strength is displayed in Figures 4(a) and 4(b), respectively. Without external magnetic field, MRSP exhibits obvious relaxation behavior especially under larger initial strain. 

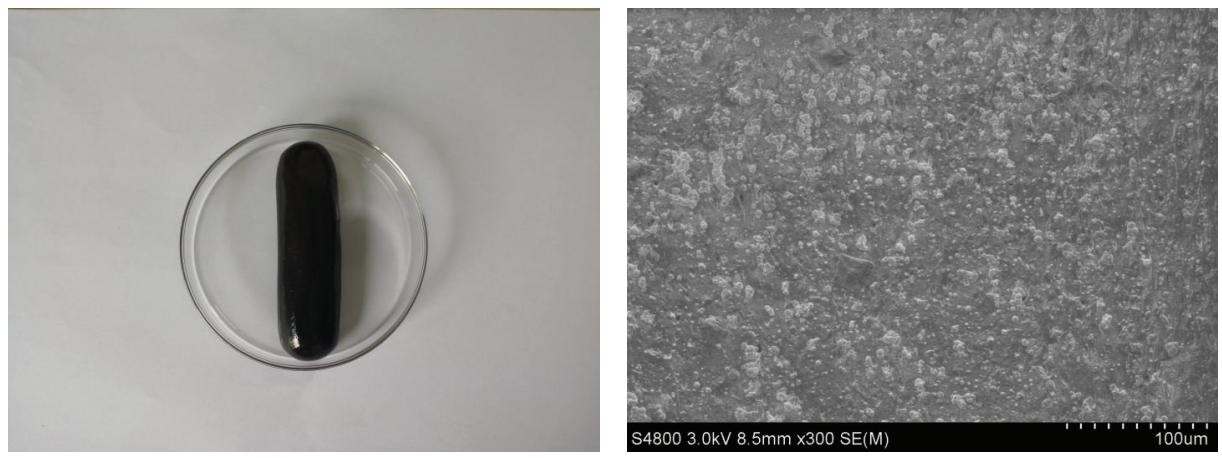

FIGURE 1: Prepared MRSP sample and SEM image of internal microstructure.

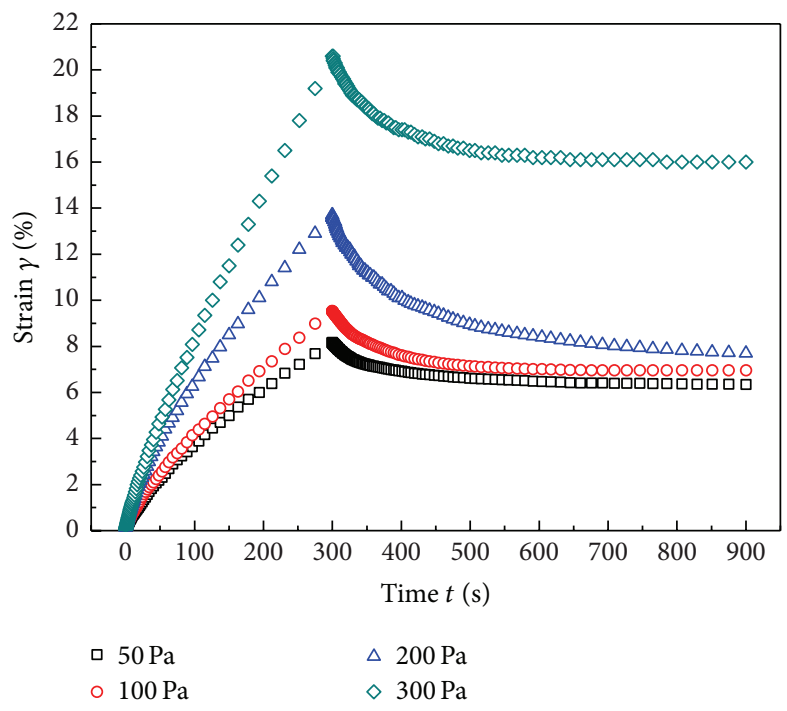

(a)

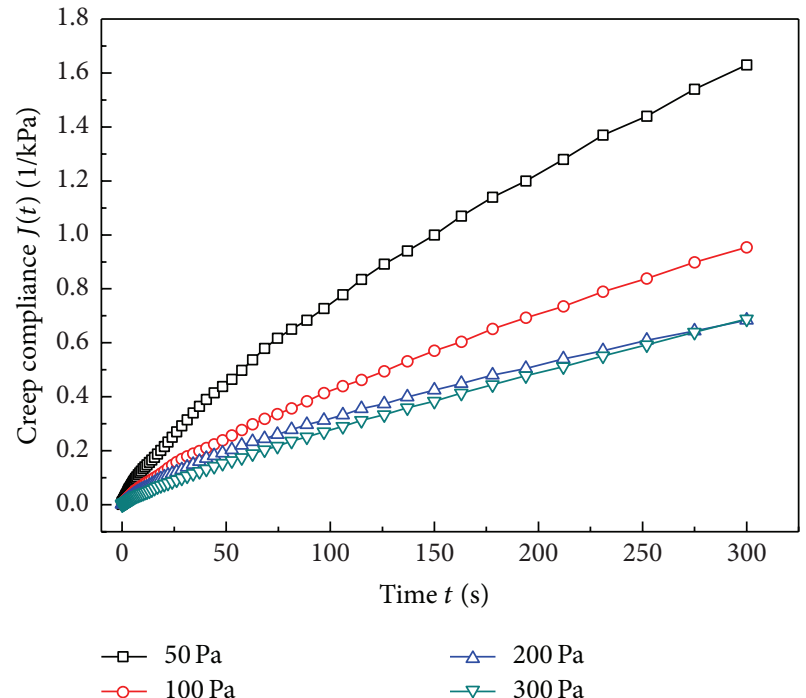

(b)

FIGURE 2: The creep characteristic of MRSP-4 on the condition of different initial stress and zero magnetic field: (a) creep and recovery phase and (b) creep compliance in creep phase.

Taking initial strain of $3.5 \%$ as example, relaxation modulus falls from $507 \mathrm{kPa}$ to $95.8 \mathrm{kPa}$ in less than one second. Besides, as shown in Figure 4(b), external magnetic field can improve relaxation modulus but delay the starting time of relaxation behavior. Interestingly, with the increasing external magnetic field strength, relaxation modulus increases with time firstly and then decreases to generate relaxation. When the external magnetic field strength reaches $156 \mathrm{kA} / \mathrm{m}$, the relaxation would not generate until time of $1 \mathrm{~s}$.

3.2. Shear Stiffening Property of MRSPs. The MRSP exhibits apparent temperature dependent property. The response of storage modulus $G^{\prime}$ to a change in shear angular frequency $\omega$ for MRSP- 4 at different temperature is shown in Figure 5. The angular frequency is applied from 1 to $100 \mathrm{rad} / \mathrm{s}$. When the angular frequency is increased, the shear rate on the MRSP also increases. As the temperature decreases, the storage modulus increases more rapidly within a certain angular frequency $(0-20 \mathrm{rad} / \mathrm{s})$ that demonstrates excellent shear stiffening property. To quantify the shear stiffening property, absolute shear stiffening effect (ASTe) and relative shear stiffening effect (RSTe) referring to characterization of magnetorheological effect [6] are introduced in (1). $G_{\max }^{\prime}$ is the maximum storage modulus of MRSP-4 induced by the shear angular frequency and $G_{\min }^{\prime}$ is the initial storage modulus. The details about MRSP-4 at different temperature are listed in Table 2:

$$
\begin{aligned}
\mathrm{ASTe} & =G_{\text {max }}^{\prime}-G_{\min }^{\prime}, \\
\mathrm{RSTe} \% & =\frac{G_{\text {max }}^{\prime}-G_{\min }^{\prime}}{G_{\min }^{\prime}} \times 100 \% .
\end{aligned}
$$

MRSP-4 displays maximum ASTe $0.805 \mathrm{MPa}$ at $10^{\circ} \mathrm{C}$ and maximum RSTe $6600 \%$ at $60^{\circ} \mathrm{C}$ as shown in Table 2. Due to the larger initial storage modulus at lower temperature, RSTe of MRSP increases with increasing temperature, but the ASTe is on the contrary.

Figure 6(a) shows the shear stiffening property of different CI contents of MRSPs at temperature $25^{\circ} \mathrm{C}$. The storage modulus of all the testing samples increases with increasing angular frequency from $0.1 \mathrm{rad} / \mathrm{s}$ to $100 \mathrm{rad} / \mathrm{s}$ which indicates that MRSPs become stiffer under application of external shear 


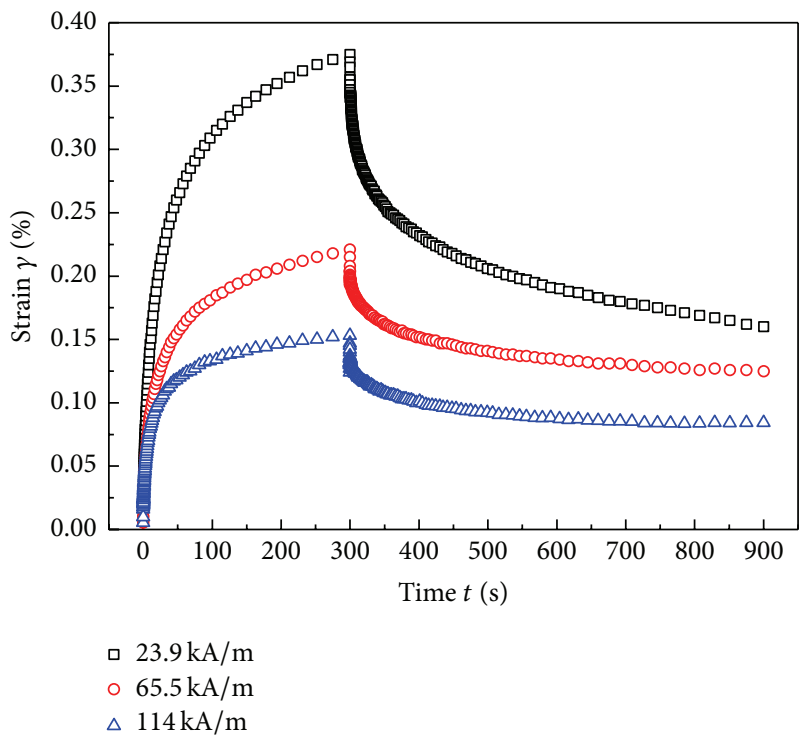

(a)

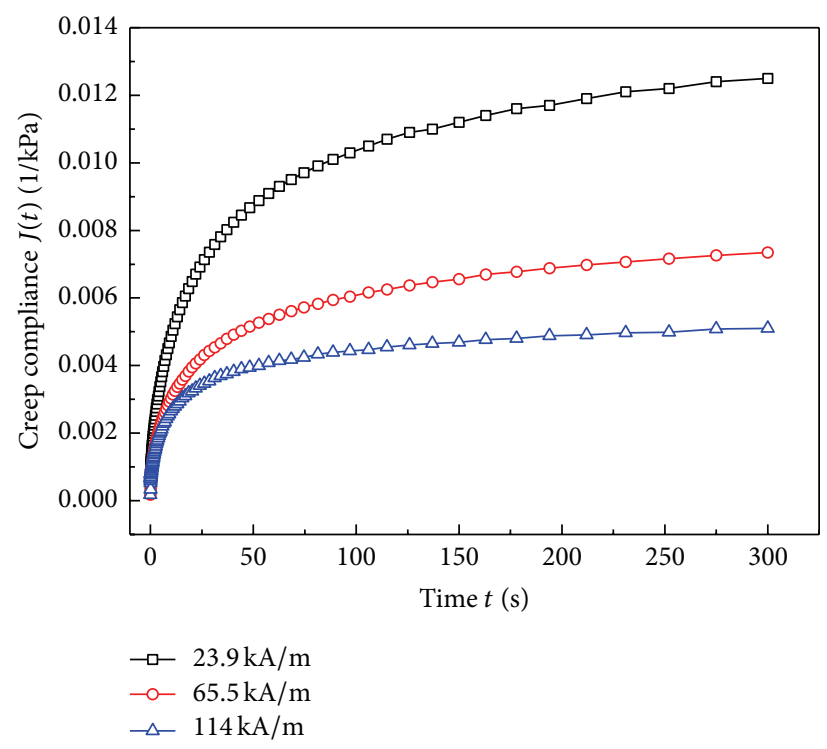

(b)

FIgURE 3: The creep characteristic of MRSP-4 on the condition of different external magnetic field strength and the same initial stress 300 Pa: (a) creep and recovery phase and (b) creep compliance in creep phase.

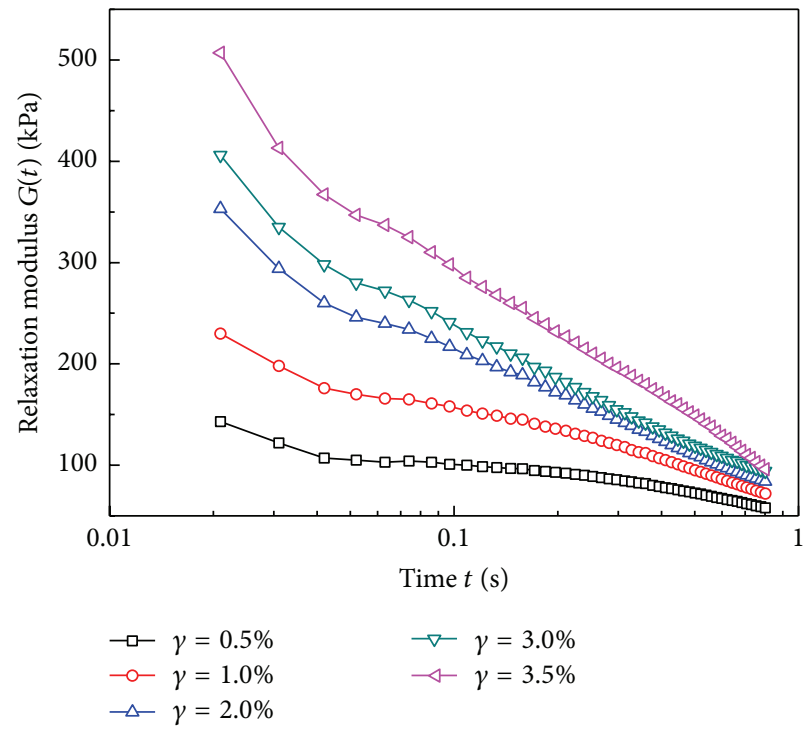

(a)

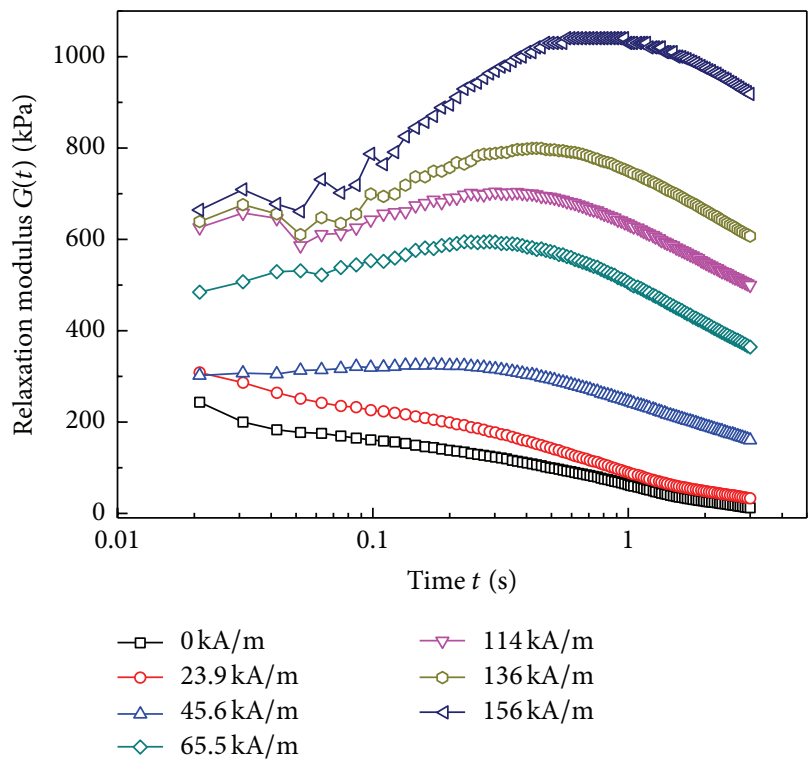

(b)

FIGURE 4: The relaxation property of MRSP-4 (a) on the condition of different initial strain (b) on the condition of different external magnetic field strength.

stress. On the condition of no external magnetic field, CI particles only play a role in reinforcing the Silly Putty matrix and enhance the storage modulus of MRSPs. The storage modulus of the MRSP- 4 as a function of angular frequency at different magnetic field strength is shown in Figure 6(b). The shear stiffening property can be controlled through the external magnetic field due to the formation of magnetic chains of
CI particles in MRSP. All the relevant details to quantify the shear stiffening effect of MRSP from Figures 6(a) and 6(b) are listed in Table 3.

Pure Silly Putty MRSP-0 displays maximum RSTe and MRSP-5 with CI volume fraction of $27.29 \%$ exhibits maximum ASTe as shown in Table 3. Due to the reinforcing effect of CI particles, more soft magnetic fillers can induce larger 
TABLE 2: $G_{\max }^{\prime}, G_{\min }^{\prime}$, ASTe, and RSTe\% of MRSP-4 in the angular frequency scanning test at different temperatures.

\begin{tabular}{lcccc}
\hline Temperature & $G_{\max }^{\prime} / \mathrm{MPa}$ & $G_{\min }^{\prime} / \mathrm{MPa}$ & $\mathrm{ASTe} / \mathrm{MPa}$ & $\mathrm{RSTe} \%$ \\
\hline $10^{\circ} \mathrm{C}$ & 0.878 & 0.073 & 0.805 & $1102.74 \%$ \\
$20^{\circ} \mathrm{C}$ & 0.594 & 0.029 & 0.565 & $1948.28 \%$ \\
$40^{\circ} \mathrm{C}$ & 0.326 & 0.006 & 0.320 & $5333.33 \%$ \\
$60^{\circ} \mathrm{C}$ & 0.201 & 0.003 & 0.198 & $6600.00 \%$ \\
\hline
\end{tabular}

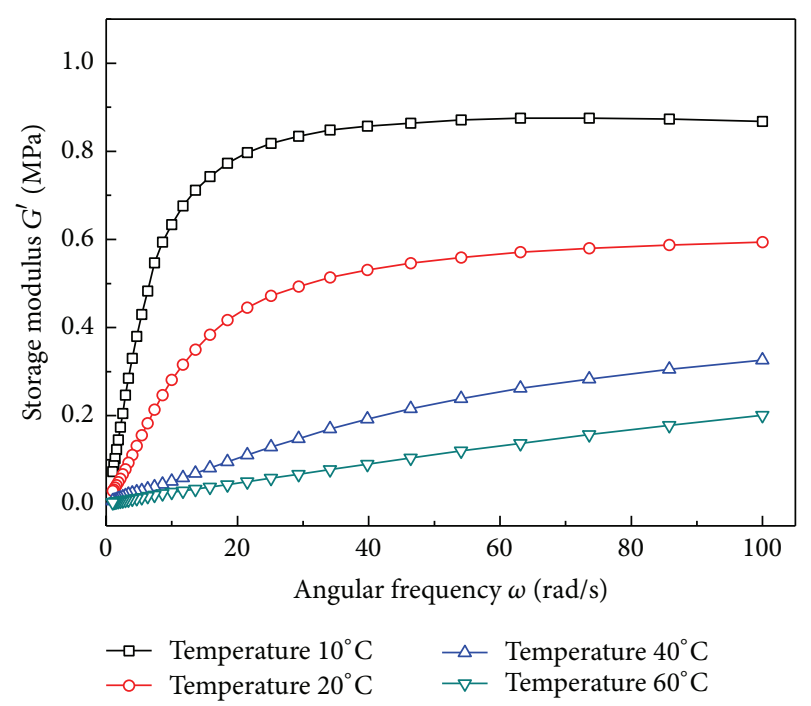

FIGURE 5: The effect of temperature on shear stiffening property for MRSP-4.

initial storage modulus at $0.1 \mathrm{rad} / \mathrm{s}$ which contribute to the descending RSTe. Meanwhile, when the external magnetic field strength is $23.9 \mathrm{kA} / \mathrm{m}$, the ASTe of MRSP-4 with CI volume fraction of $20.8 \%$ reaches to $1.01 \mathrm{MPa}$. But as the magnetic field strength is increased, both ASTe and RSTe decrease. Therefore, it can be concluded that the state of larger magnetic field strength is not conducive to the shear stiffening property of MRSP, but lower magnetic field strength can improve ASTe of MRSP.

Overall, MRSP is a dilatant material where the viscosity increases faster than the strain rate. There are two mechanisms (and hence two characteristic time scales) at work in this material $[1,12]$. The high molecular weight PDMS has a characteristic polymeric relaxation time $\lambda_{\text {relax }}$. However due to the boric acid there are also transient boron mediated "crosslinks" arising from associating boron linkages. These act to give the MRSP a behavior more like an elastic solid than a liquid. However since these "crosslinks" are dynamic (with a characteristic time $\lambda_{\text {assoc }}$, i.e., much shorter than $\lambda_{\text {relax }}$ ) the material is not permanently locked in place and can consequently flow under the correct conditions. Therefore at time scales longer than $\lambda_{\text {assoc }}$ the MRSP behaves like a high molecular weight polymeric fluid (with a characteristic relaxation of $\lambda_{\text {relax }}$ ). Over time scales much shorter than $\lambda_{\text {assoc }}$ MRSP behaves like a crosslinked elastic solid.

The mechanisms of shear stiffening behind MRSPs are very complex, but our goal here is to discover a relatively simple function to accurately fit the storage modulus versus angular frequency at different temperature, CI contents, and external magnetic field strength. Galindo-Rosales et al. [13, 14] used an apparent viscosity function with 11 parameters to describe the three characteristic regions of shear thickening fluid (STF), including the slight shear thinning at low shear rates, followed by a sharp increase in viscosity over the critical shear rate, and a subsequent, but pronounced, shear thinning region at high shear rates. Furthermore, Tian et al. [15] proposed a modified model shown in (2) to overcome the drawback of 11 parameters function that if any $n_{1}, n_{2}$, or $n_{3}$ was a decimal, the value in the bracket must be positive; otherwise the equation failed:

$$
\eta(\dot{\gamma})= \begin{cases}\eta_{1}(\dot{\gamma})=\eta_{c}+\frac{\eta_{0}-\eta_{c}}{1+\left|\left[K_{1}\left(\dot{\gamma}^{2} /\left(\dot{\gamma}-\dot{\gamma}_{c}\right)\right)\right]\right|^{n_{1}},} & \dot{\gamma} \leq \dot{\gamma}_{c}, \\ \eta_{2}(\dot{\gamma})=\eta_{\max }+\frac{\eta_{c}-\eta_{\max }}{1+\left|\left[K_{2}\left(\left(\dot{\gamma}-\dot{\gamma}_{c}\right) /\left(\dot{\gamma}-\dot{\gamma}_{\max }\right)\right) \dot{\gamma}\right]\right|^{n_{2}}}, & \dot{\gamma}_{c} \leq \dot{\gamma} \leq \dot{\gamma}_{\max }, \\ \eta_{3}(\dot{\gamma})=\frac{\eta_{\max }}{1+\left|\left[K_{3}\left(\dot{\gamma}-\dot{\gamma}_{\max }\right)\right]\right|^{n_{3}},} & \dot{\gamma}_{\max } \leq \dot{\gamma} .\end{cases}
$$

A new function is proposed in (3) through imitating part of (2) to describe the shear stiffening property of MRSPs. In (3), $G_{\min }^{\prime}$ represents the storage modulus at initial experimental angular frequency $\omega_{\min } ; G_{\max }^{\prime}$ represents the maximum storage modulus at final experimental angular frequency $\omega_{\max }$; parameter $n$ refers to the slope of the shear stiffening behavior in log-log plot; parameter $K$ is responsible for the ratio of changed storage modulus induced by frequency to the initial storage modulus (RSTe); finally, parameter $A$ is introduced to overcome the shortcoming that the final experimental point $\left(\omega_{\max }, G_{\max }^{\prime}\right)$ in curves cannot be fitted:

$$
\begin{aligned}
& G^{\prime}(\omega) \\
& =G_{\max }^{\prime} \\
& \quad-\frac{G_{\max }^{\prime}-G_{\min }^{\prime}}{1+\left|\left[K\left(\left(A \omega-\omega_{\min }\right) /\left(A \omega-\omega_{\max }\right)\right) \omega\right]\right|^{n}} .
\end{aligned}
$$


TABLE 3: $G_{\max }^{\prime}, G_{\min }^{\prime}$, ASTe, and RSTe\% of MRSPs obtained from Figure 6.

\begin{tabular}{lcccc}
\hline Samples & $G_{\max }^{\prime} / \mathrm{MPa}$ & $G_{\min }^{\prime} / \mathrm{MPa}$ & ASTe/MPa & RSTe\% \\
\hline MRSP-0 & 0.369 & 0.000475 & 0.368525 & $77584.21 \%$ \\
MRSP-1 & 0.439 & 0.001180 & 0.437820 & $37103.39 \%$ \\
MRSP-2 & 0.486 & 0.001800 & 0.484200 & $26900.00 \%$ \\
MRSP-3 & 0.556 & 0.002840 & 0.553160 & $19477.46 \%$ \\
MRSP-4, 0 kA/m & 0.617 & 0.014200 & 0.602800 & $4245.07 \%$ \\
MRSP-4, 23.9 kA/m & 1.30 & 0.29 & 1.01 & $348.28 \%$ \\
MRSP-4, 65.5 kA/m & 1.75 & 1.07 & 0.68 & $63.55 \%$ \\
MRSP-4, 114 kA/m & 1.87 & 1.28 & 0.59 & $46.09 \%$ \\
MRSP-5 & 0.658 & 0.043500 & 0.614500 & $1412.64 \%$ \\
\hline
\end{tabular}

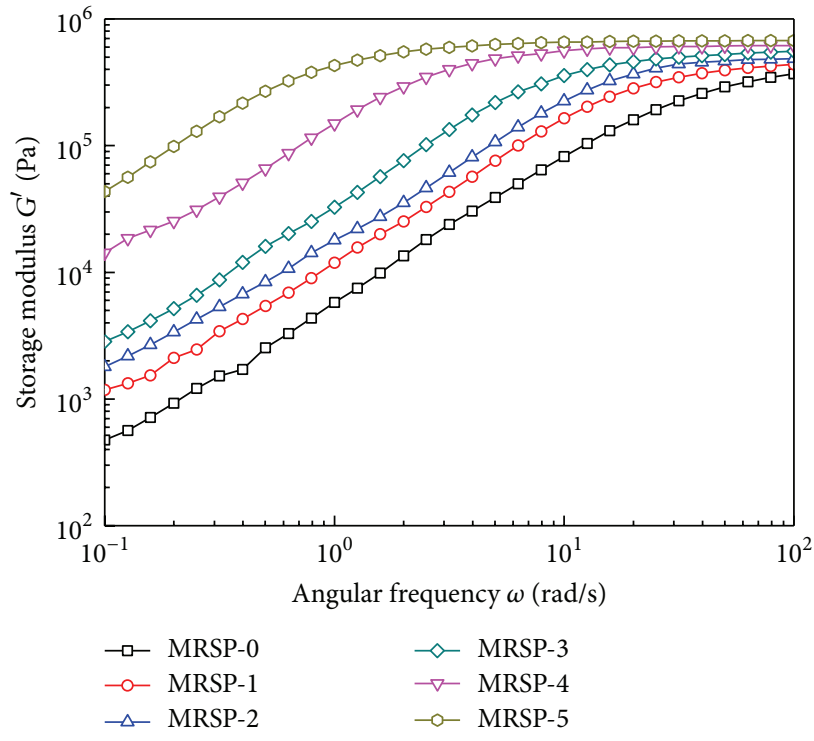

(a)

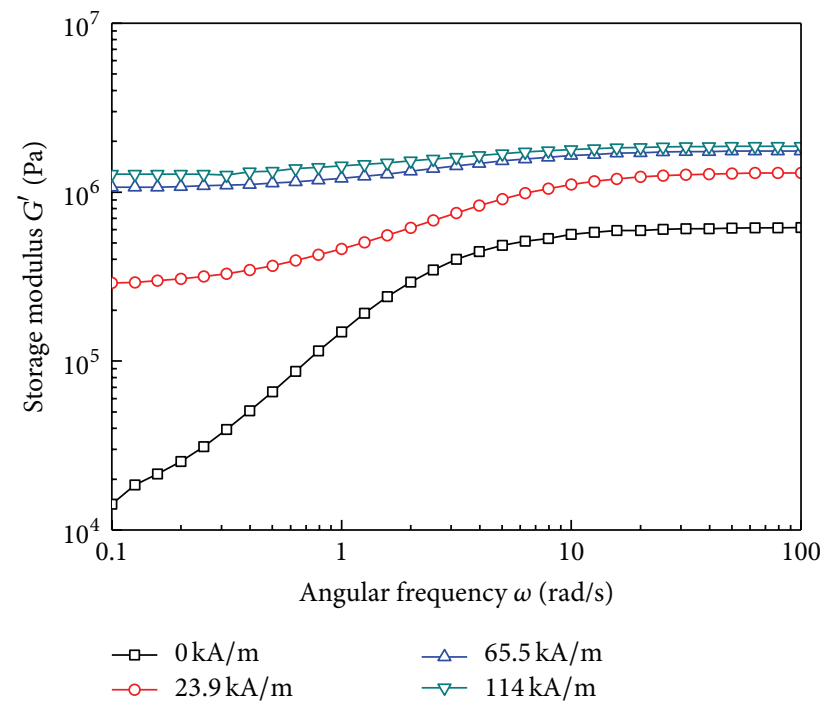

(b)

Figure 6: The storage modulus of MRSPs as a function of angular frequency at $25^{\circ} \mathrm{C}$ (a) different CI contents and (b) different external magnetic field strength for MRSP-4.

Figure 7 shows the fitting curves of all the angular frequency scanning testing data on the condition of different temperature, CI contents, and external magnetic field strength. It is obvious that (3) fits to the experimental results perfectly. Levenberg-Marquardt algorithm (LMA) is applied in all the fitting procedures. This algorithm combines the Gauss-Newton and steepest descent methods to obtain the values of the parameters by an iterative chi-square minimisation technique. Fitting is considered to have converged when the difference between the $X^{2}$ values obtained in two successive iterations is smaller than a given tolerance, chosen here as $10^{-10}$

Table 4 lists all the fitting values of parameters in (3). It is observed from related coefficient $R$ that a high fitting precision is obtained in each fitting result. From each section, parameter $K$ is inversely proportional to the value of RSTe; interestingly, when there is an external magnetic field applied (Section III), due to higher initial storage modulus and lower changed storage modulus, parameter $K$ increases with the value magnetic field strength obviously. Parameter $n$ reflects the slope of shear stiffening behavior. In log-log plot, the slope difference in each section is not very distinct which leads to tiny change or even the same values of parameter $n$. Besides, parameter $A$ is relatively independent of experimental data that means the value changes a little in each section.

3.3. Magnetorheological Property of MRSPs. MRSP is also a novel magnetorheological material whose mechanical properties are highly dependent on the externally applied magnetic field. Figure 8 shows the response of storage modulus to a change in magnetic flux density for MRSP-4 at different temperature. The angular frequency applied on MRSP-4 is $10 \mathrm{rad} / \mathrm{s}$ and magnetic flux density increases from $0 \mathrm{~T}$ to $0.894 \mathrm{~T}$. Similar to its shear stiffening property, magnetorheological effect of MRSP is also influenced by temperature. Meanwhile, absolute magnetorheological effect (AMRe) and relative magnetorheological effect (RMRe) are introduced in (4) to quantify the magnetorheological property. $G_{\max }^{\prime}$ is the maximum storage modulus of MRSP- 4 induced by the external magnetic field and $G_{\min }^{\prime}$ is the initial storage 
TABLE 4: All the fitting values of parameters.

\begin{tabular}{|c|c|c|c|c|c|c|c|}
\hline Section & Samples & Experimental condition & RSTe\% & $K$ & $A$ & $n$ & $R$ \\
\hline \multirow{4}{*}{ Section I } & \multirow{4}{*}{ MRSP-4 } & $10^{\circ} \mathrm{C}, 1-100 \mathrm{rad} / \mathrm{s}$ & $1102.74 \%$ & 2.479 & 1.20025 & 0.801 & 0.99982 \\
\hline & & $20^{\circ} \mathrm{C}, 1-100 \mathrm{rad} / \mathrm{s}$ & $1948.28 \%$ & 0.778 & 0.95077 & 0.729 & 0.99998 \\
\hline & & $40^{\circ} \mathrm{C}, 1-100 \mathrm{rad} / \mathrm{s}$ & $5333.33 \%$ & 0.064 & 1.00956 & 0.675 & 0.99961 \\
\hline & & $60^{\circ} \mathrm{C}, 1-100 \mathrm{rad} / \mathrm{s}$ & $6600.00 \%$ & 0.029 & 1.00036 & 0.624 & 0.99902 \\
\hline \multirow{6}{*}{ Section II } & MRSP-0 & $25^{\circ} \mathrm{C}, 0.1-100 \mathrm{rad} / \mathrm{s}$ & $77584.21 \%$ & 0.164 & 0.902 & 0.735 & 0.99942 \\
\hline & MRSP-1 & $25^{\circ} \mathrm{C}, 0.1-100 \mathrm{rad} / \mathrm{s}$ & $37103.39 \%$ & 0.503 & 0.902 & 0.735 & 0.99994 \\
\hline & MRSP-2 & $25^{\circ} \mathrm{C}, 0.1-100 \mathrm{rad} / \mathrm{s}$ & $26900.00 \%$ & 0.903 & 0.902 & 0.735 & 0.99926 \\
\hline & MRSP-3 & $25^{\circ} \mathrm{C}, 0.1-100 \mathrm{rad} / \mathrm{s}$ & $19477.46 \%$ & 2.210 & 0.902 & 0.735 & 0.99985 \\
\hline & MRSP-4 & $25^{\circ} \mathrm{C}, 0.1-100 \mathrm{rad} / \mathrm{s}$ & $4245.07 \%$ & 23.459 & 0.902 & 0.735 & 0.99993 \\
\hline & MRSP-5 & $25^{\circ} \mathrm{C}, 0.1-100 \mathrm{rad} / \mathrm{s}$ & $1412.64 \%$ & 227.545 & 0.902 & 0.735 & 0.99959 \\
\hline \multirow{3}{*}{ Section III } & \multirow{3}{*}{ MRSP-4 } & $0.1-100 \mathrm{rad} / \mathrm{s}, 23.9 \mathrm{kA} / \mathrm{m}$ & $348.28 \%$ & 18.044 & 0.5 & 0.621 & 0.99956 \\
\hline & & $0.1-100 \mathrm{rad} / \mathrm{s}, 65.5 \mathrm{kA} / \mathrm{m}$ & $63.55 \%$ & 26.951 & 0.5 & 0.621 & 0.99966 \\
\hline & & $0.1-100 \mathrm{rad} / \mathrm{s}, 114 \mathrm{kA} / \mathrm{m}$ & $46.09 \%$ & 34.681 & 0.5 & 0.621 & 0.99876 \\
\hline
\end{tabular}

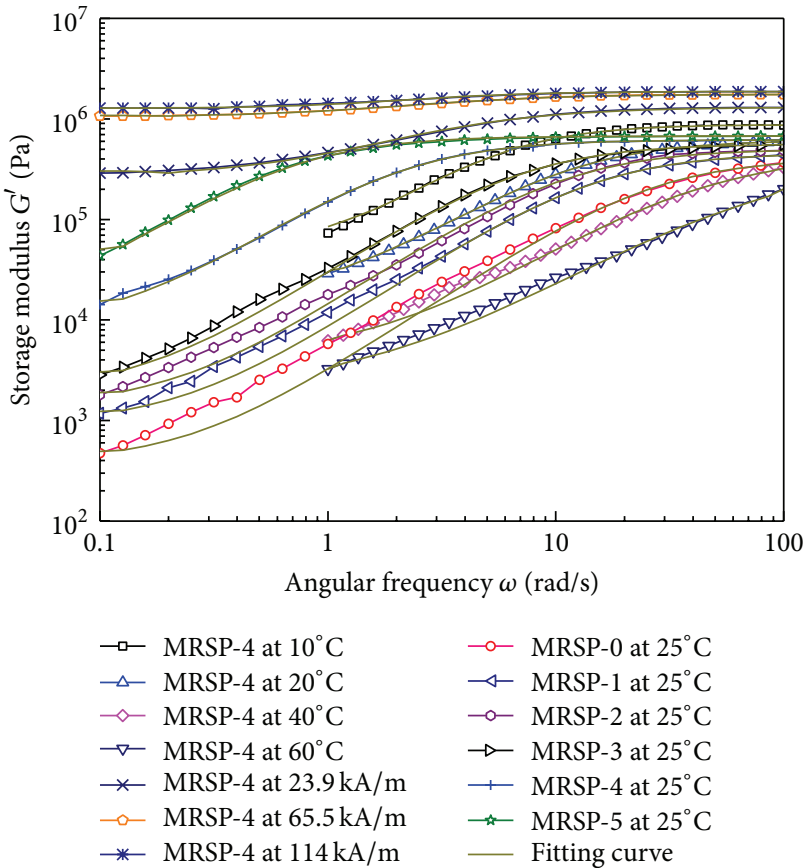

FIGURE 7: Fitting curves of all the experimental data.

modulus. The details obtained from Figure 8 are listed in Table 5:

$$
\begin{aligned}
\text { AMRe } & =G_{\text {max }}^{\prime}-G_{\text {min }}^{\prime}, \\
\operatorname{RMRe} \% & =\frac{G_{\max }^{\prime}-G_{\min }^{\prime}}{G_{\min }^{\prime}} \times 100 \% .
\end{aligned}
$$

The overall trends displayed in Table 5 are similar to those presented in Table 2. When the temperature is increased, RMRe of MRSP-4 enhances significantly, but the AMRe is on the contrary. Higher temperature can soften the Silly Putty matrix, thus inducing the lower initial storage modulus and less resistance to the regular arrangement of CI particles.
TABLE 5: $G_{\max }^{\prime}, G_{\min }^{\prime}$, AMRe, and RMRe\% of MRSP-4 with externally applied magnetic field at different temperatures.

\begin{tabular}{lcccc}
\hline Temperature & $G_{\max }^{\prime} / \mathrm{MPa}$ & $G_{\min }^{\prime} / \mathrm{MPa}$ & $\mathrm{AMRe} / \mathrm{MPa}$ & $\mathrm{RMRe} \%$ \\
\hline $10^{\circ} \mathrm{C}$ & 2.17 & 0.754 & 1.416 & $187.80 \%$ \\
$20^{\circ} \mathrm{C}$ & 1.59 & 0.422 & 1.168 & $276.78 \%$ \\
$40^{\circ} \mathrm{C}$ & 1.25 & 0.109 & 1.141 & $1046.79 \%$ \\
$60^{\circ} \mathrm{C}$ & 1.01 & 0.00242 & 1.00758 & $41635.54 \%$ \\
\hline
\end{tabular}

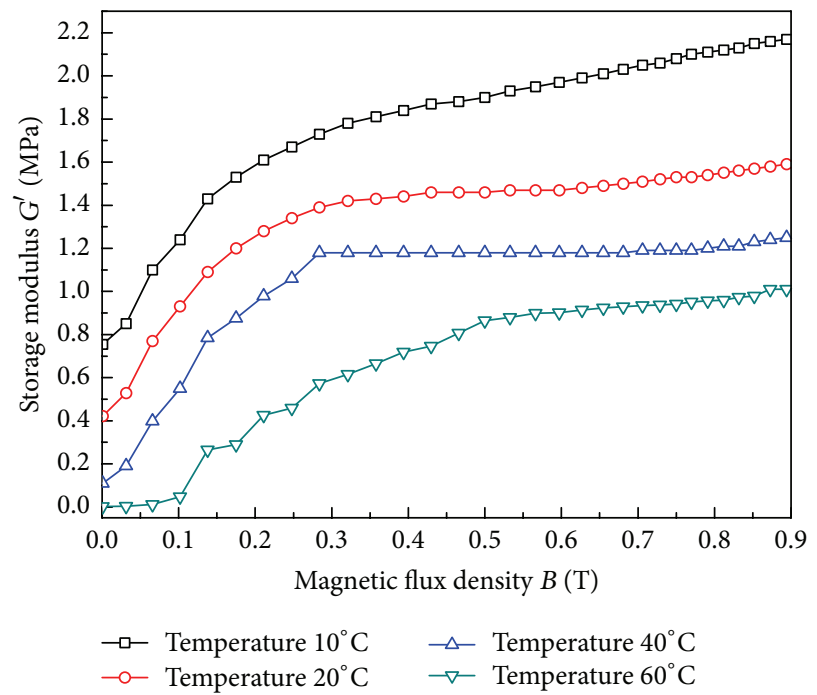

FIGURE 8: The effect of temperature on magnetorheological property for MRSP-4.

Figures 9(a) and 9(b) show the magnetic field-dependent storage modulus of MRSPs with different CI contents at angular frequency $20 \mathrm{rad} / \mathrm{s}$ and $100 \mathrm{rad} / \mathrm{s}$, respectively. These experimental curves demonstrate that there is a tendency for the storage modulus of MRSPs to increase with the increase of the magnetic flux density firstly and then saturate with further increases of the magnetic flux density. CI content is observed from Table 6 to have direct influence on AMRe of MRSPs, 
TABLE 6: $G_{\max }^{\prime}, G_{\min }^{\prime}$, AMRe, and RMRe\% of MRSPs with externally applied magnetic field at different angular frequencies.

\begin{tabular}{|c|c|c|c|c|c|}
\hline Angular frequency & Samples & $G_{\max }^{\prime} / \mathrm{MPa}$ & $G_{\min }^{\prime} / \mathrm{MPa}$ & AMRe/MPa & RMRe\% \\
\hline \multirow{5}{*}{$20 \mathrm{rad} / \mathrm{s}$} & MRSP-1 & 0.485 & 0.356 & 0.129 & $36.23 \%$ \\
\hline & MRSP-2 & 0.686 & 0.400 & 0.286 & $71.5 \%$ \\
\hline & MRSP-3 & 1.61 & 0.652 & 0.958 & $146.93 \%$ \\
\hline & MRSP-4 & 2.36 & 0.746 & 1.614 & $216.35 \%$ \\
\hline & MRSP-5 & 2.75 & 1.08 & 1.670 & $154.63 \%$ \\
\hline \multirow{5}{*}{$100 \mathrm{rad} / \mathrm{s}$} & MRSP-1 & 0.775 & 0.543 & 0.232 & $42.73 \%$ \\
\hline & MRSP-2 & 1.01 & 0.680 & 0.330 & $48.53 \%$ \\
\hline & MRSP-3 & 1.58 & 0.886 & 0.694 & $78.33 \%$ \\
\hline & MRSP-4 & 1.97 & 1.000 & 0.970 & $97 \%$ \\
\hline & MRSP-5 & 2.96 & 1.450 & 1.510 & $104.14 \%$ \\
\hline
\end{tabular}

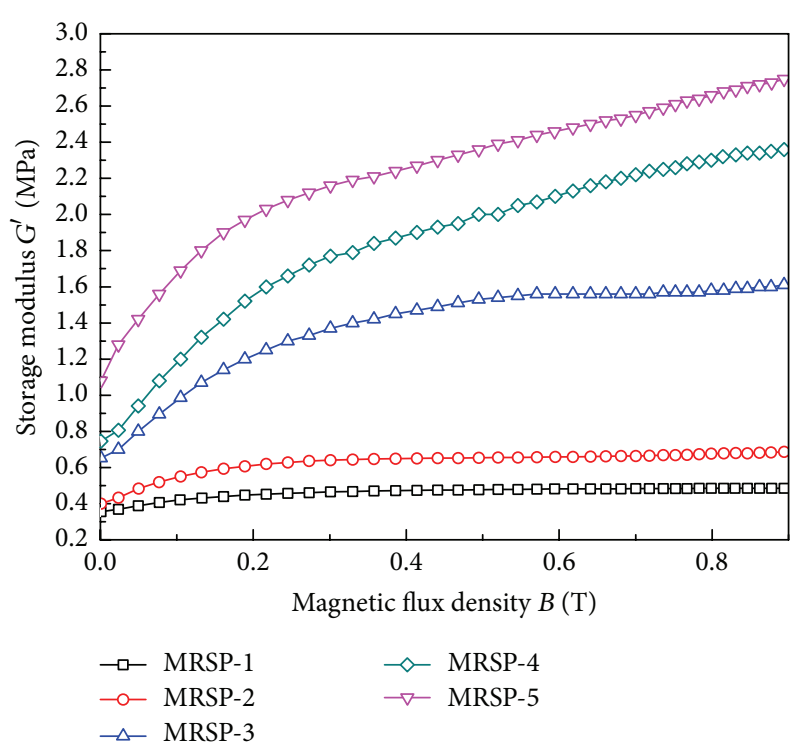

(a)

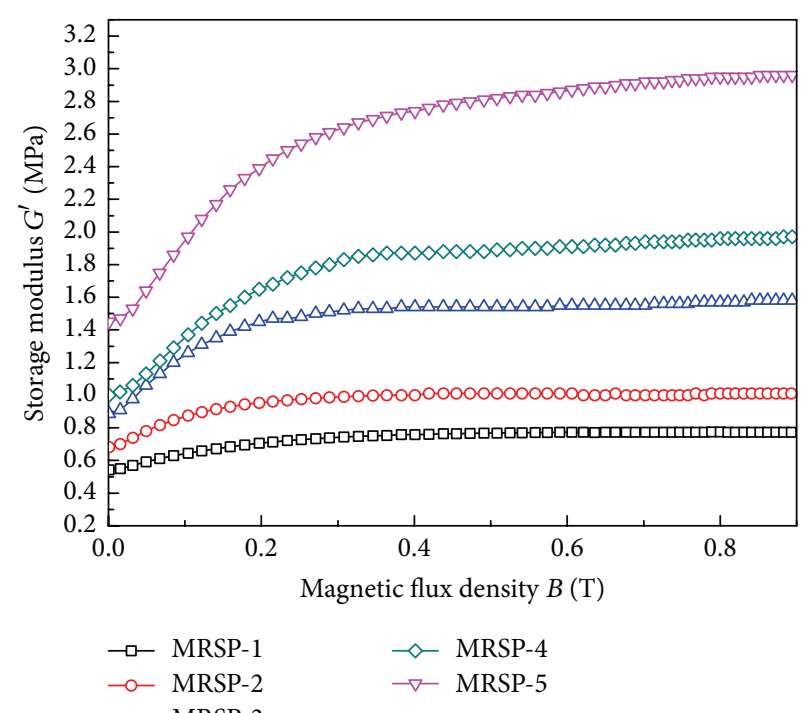

(b)

FIGURE 9: The storage modulus of MRSPs as a function of magnetic flux density at $25^{\circ} \mathrm{C}$ (a) angular frequency $20 \mathrm{rad} / \mathrm{s}$ and (b) angular frequency $100 \mathrm{rad} / \mathrm{s}$.

which means that higher CI content induces greater AMRe. The magnetoinduced storage modulus is mainly attributed to the particle chains formed under external magnetic field. However, greater AMRe is not certain to keep up with higher RMRe. For example, when the applied angular frequency is $20 \mathrm{rad} / \mathrm{s}$, AMRe of MRSP-4 is $1.614 \mathrm{MPa}$ less than that of MRSP-5, but RMRe of MRSP-4 reaches to maximum value of $216.35 \%$. Moreover, it can also be concluded that magnetorheological effect of MRSPs on lower shear rate state is greater than that of higher shear rate state.

3.4. Modelling on Magnetoinduced Behavior. Before applying external magnetic field, CI particles are homogeneously dispersed in Silly Putty matrix, but when there is an external magnetic field applied, particles are arranged in chain structure instantaneously as shown in Figure 10. In the chain structure, CI particles are simplified to spherical particles with the radius $R$ and the additional acting force between two particles caused by magnetic field leads to the increment of the storage modulus. However, the viscosity of Silly Putty matrix is much larger than general liquids which can cause tremendous obstruction to the formation of pulling-through particle chains. So a nonperforative particle aggregated chains model is displayed in Figure 10 to explain the magnetoinduced mechanism. In the model, MRSP is divided into several square columns with the length of side $2 R$ and the height $h$. At zero magnetic field strength, particles are randomly dispersed in each square column and MRSP exhibits isotropy. When a low magnetic field is applied, particles aggregate to several apart chains along the direction of magnetic field in each square column with different length $d_{i}$ and the total length of the chains included in a square column can be marked as $\sum d_{i}$. The chains in parallel are sparse due to the low magnetic field strength. However, when the magnetic field strength increases, the aggregated chains in each square column turn unstable and a small disturbance (shearing) may drift some particles in a square column away to aggregate to another apart chain in a new square column. So the chains in parallel 


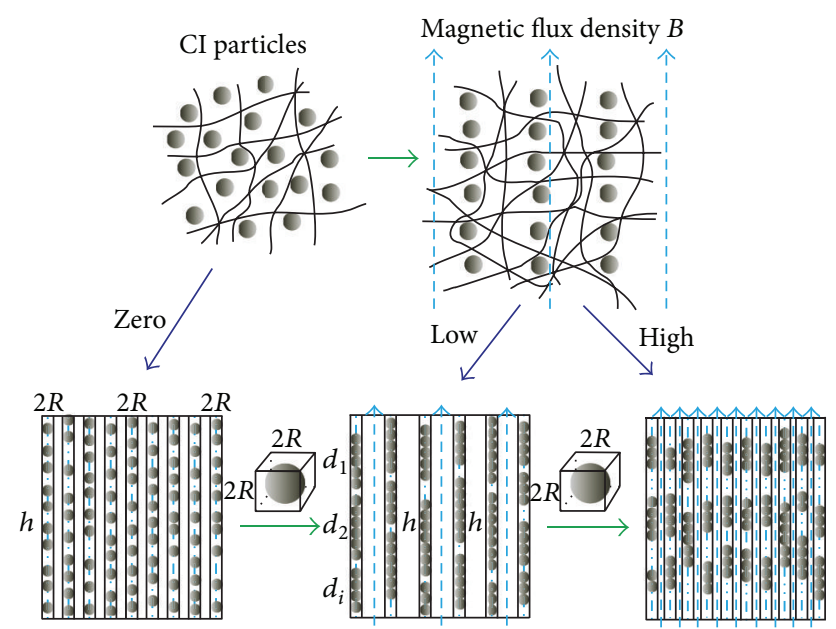

FIGURE 10: Schematic diagram of chain-formation mechanism for MRSPs under magnetic field and the nonperforative particle aggregated chains model.

become close and even to form particle columns extremely, but the total chains length in a square column becomes short.

For the square columns, the effective permeability $\mu_{\text {sceff }}$ can be calculated by using Maxwell Garnett mixing rule as $[16,17]$

$$
\mu_{\mathrm{sceff}}=\mu_{\mathrm{CI}}+2 \phi \mu_{\mathrm{SP}} \frac{\mu_{\mathrm{CI}}-\mu_{\mathrm{SP}}}{\mu_{\mathrm{CI}}+\mu_{\mathrm{SP}}-\phi\left(\mu_{\mathrm{CI}}-\mu_{\mathrm{SP}}\right)},
$$

where $\mu_{\mathrm{CI}}$ and $\mu_{\mathrm{SP}}$ are the relative permeability of CI particles and Silly Putty matrix, respectively. $\phi$ is the particles volume fraction in each square column. In the granular basic unit, the volume fraction $f$ can be calculated as $f=(4 / 3) \pi R^{3} /(2 R)^{3}=$ $\pi / 6$. So $\phi$ can be expressed as below:

$$
\phi=\frac{\sum d_{i}}{h} f=\frac{\pi \sum d_{i}}{6 h}
$$

where $\sum d_{i} / h$ is relevant to external magnetic field strength.

Besides, if $\phi_{\mathrm{sc}}$ is the volume fraction of the square columns in MRSP and can be expressed as

$$
\phi_{\mathrm{sc}}=\frac{\phi_{p}}{\phi}=\frac{6 h \phi_{p}}{\pi \sum d_{i}}
$$

where $\phi_{p}$ is the particles volume fraction in MRSP.

So, the effective permeability $\mu_{\text {eff }}$ along the direction of square columns can be calculated as follows according to the parallel connection rule $[18,19]$ and (5)-(7):

$$
\begin{aligned}
\mu_{\mathrm{eff}}= & \mu_{\mathrm{sceff}} \phi_{\mathrm{sc}}+\mu_{\mathrm{SP}}\left(1-\phi_{\mathrm{sc}}\right) \\
= & \mu_{\mathrm{SP}} \\
& +2 \phi_{p} \mu_{\mathrm{SP}} \frac{\mu_{\mathrm{CI}}-\mu_{\mathrm{SP}}}{\mu_{\mathrm{CI}}+\mu_{\mathrm{SP}}-\left(\pi \sum d_{i} / 6 h\right)\left(\mu_{\mathrm{CI}}-\mu_{\mathrm{SP}}\right)} .
\end{aligned}
$$

When shear strain is applied on MRSP under the external magnetic field, the relative permeability of MRSP changes which can induce the generation of additional force, that is, magnetoinduced shear stress. The magnetoinduced shear stress can be expressed as

$$
\tau_{m}=-\frac{1}{2} \mu_{0} \frac{\partial \mu_{\mathrm{eff}}}{\partial \gamma} H_{0}^{2}
$$

where $\mu_{0}$ is the vacuum permeability, $\gamma$ is the shear strain, and $H_{0}$ is the external magnetic field. The shear strain can be marked as

$$
\gamma=\frac{\Delta}{h}
$$

The magnetoinduced shear stress can be obtained by substituting (8) and (10) into (9) as

$$
\begin{aligned}
\tau_{m}= & \frac{\pi \sum d_{i}}{6 h} \cdot \frac{\mu_{0} \mu_{\mathrm{SP}} \phi_{p}}{\gamma} \\
& \cdot \frac{\left(\mu_{\mathrm{CI}}-\mu_{\mathrm{SP}}\right)^{2}}{\left[\mu_{\mathrm{CI}}+\mu_{\mathrm{SP}}-\left(\pi \sum d_{i} / 6 h\right)\left(\mu_{\mathrm{CI}}-\mu_{\mathrm{SP}}\right)\right]^{2}} H_{0}^{2} .
\end{aligned}
$$

The magnetoinduced shear modulus can be calculated as

$$
\begin{aligned}
\Delta G= & \frac{\tau_{m}}{\gamma} \\
= & \frac{\pi \sum d_{i}}{6 h} \cdot \frac{\mu_{0} \mu_{\mathrm{SP}} \phi_{p}}{\gamma^{2}} \\
& \cdot \frac{\left(\mu_{\mathrm{CI}}-\mu_{\mathrm{SP}}\right)^{2}}{\left[\mu_{\mathrm{CI}}+\mu_{\mathrm{SP}}-\left(\pi \sum d_{i} / 6 h\right)\left(\mu_{\mathrm{CI}}-\mu_{\mathrm{SP}}\right)\right]^{2}} H_{0}^{2} .
\end{aligned}
$$

Therefore, the storage modulus $G^{\prime}$ can be calculated as below:

$$
\begin{aligned}
G^{\prime}= & G_{\min }^{\prime}+\Delta G^{\prime} \\
= & G_{\min }^{\prime}+\frac{\pi \sum d_{i}}{6 h} \cdot \frac{\mu_{0} \mu_{\mathrm{SP}} \phi_{p}}{\gamma^{2}} \\
& \cdot \frac{\left(\mu_{\mathrm{CI}}-\mu_{\mathrm{SP}}\right)^{2}}{\left[\mu_{\mathrm{CI}}+\mu_{\mathrm{SP}}-\left(\pi \sum d_{i} / 6 h\right)\left(\mu_{\mathrm{CI}}-\mu_{\mathrm{SP}}\right)\right]^{2}} H_{0}^{2} .
\end{aligned}
$$

Furthermore, the relative permeability of CI particles $\mu_{\mathrm{CI}}$ is the function of external magnetic field strength, which can be obtained from Frohlich-Kennelly equation [20]:

$$
\mu_{\mathrm{CI}}\left(H_{0}\right)=1+\frac{\left(\mu^{0}-1\right) M_{s} / H_{0}}{\left(\mu^{0}-1\right)+M_{s} / H_{0}},
$$

where $\mu^{0}$ is the relative permeability of CI particles at zerofield strength and $M_{s}$ is the magnitude of the saturation magnetization.

Besides, the variable $\sum d_{i} / h$ can be seen as the function of external magnetic field strength, and the possible function can be expressed as below, and $a, b$, and $c$ are the parameters:

$$
\frac{\sum d_{i}}{h}=a+b \cdot e^{c \cdot H_{0}} .
$$




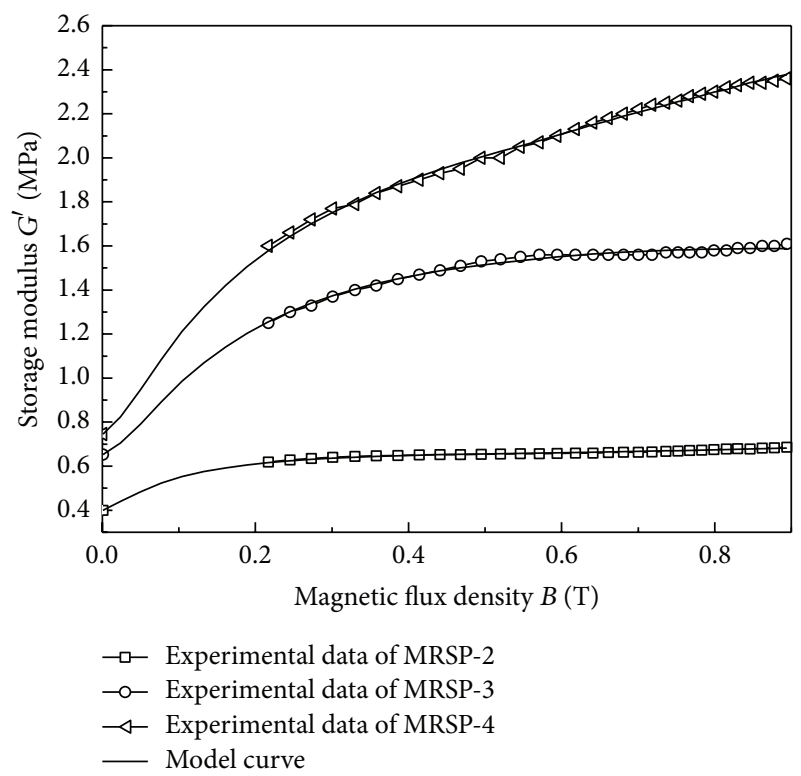

(a)

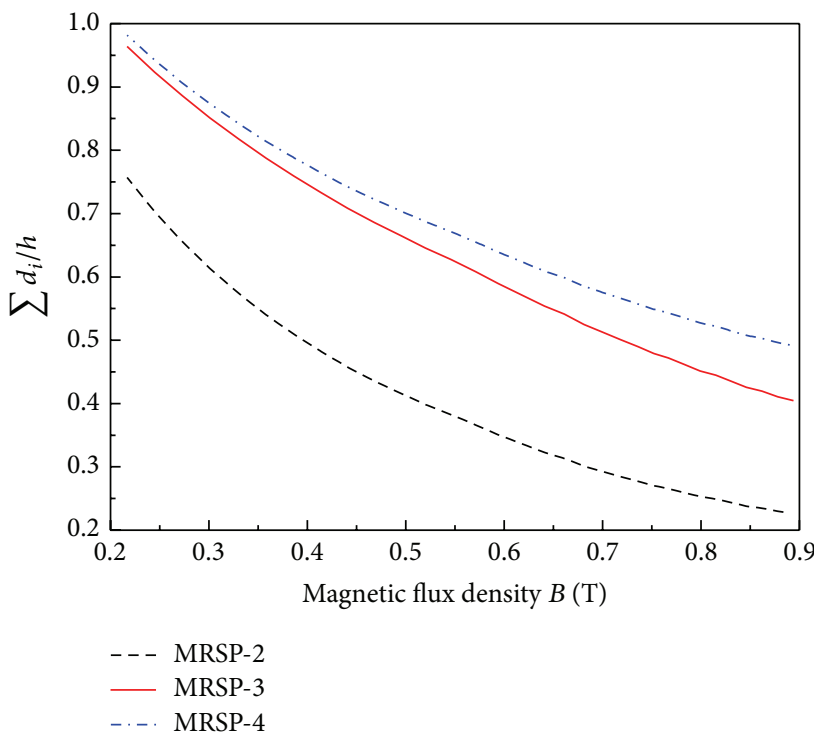

(b)

FIGURE 11: (a) $G^{\prime}-B$ curves of MRSPs based on the nonperforative particle aggregated chains model. (b) The trend of variable $\sum d_{i} / h$ with magnetic flux density $B$.

From (13) to (15), the vacuum permeability $\mu_{0}$ is $4 \pi \times$ $10^{-7} \mathrm{H} / \mathrm{m}$ and the relative permeability of Silly Putty $\mu_{\mathrm{SP}}$ is 1. Besides, for CI particles, the relative permeability at zerofield strength $\mu^{0}$ is 70 and the magnitude of the saturation magnetization $M_{s}$ reaches $1360 \mathrm{kA} / \mathrm{m}$ [21]. Furthermore, the applied shear strain $\gamma$ is $0.1 \%$. Therefore, according to the nonperforative particle aggregated chains model, taking MRSP2 , MRSP-3, and MRSP-4 for example ( $\omega=20 \mathrm{rad} / \mathrm{s})$, the $G^{\prime}-B$ curves are displayed in Figure 11(a) comparing with the experimental data. The model curves agree well with experimental data which indicates that single-chain aggregation model can describe magnetoinduced mechanical behavior well. From Figure 11(a), the variation trend of $\sum d_{i} / h$ as a function of magnetic field strength is shown in Figure 11(b). It is obvious that larger particle volume fraction induces larger value of $\sum d_{i} / h$ due to more particles in each square column. However, variable $\sum d_{i} / h$ is a decreasing function of magnetic field strength which is in consistency with the description in the front.

\section{Conclusions}

In this work, the multifunctional composite MRSPs with shear stiffening property and magnetorheological property were prepared. Both static mechanical behaviors including creep and relaxation and dynamic rheological behavior were investigated by rheometer. Firstly, MRSPs exhibited obvious creep and relaxation behavior, but a low external magnetic field strength $(23.9 \mathrm{kA} / \mathrm{m})$ could substantially confine creep deformation and creep compliance of MRSPs (strain of MRSP-4 from $20.6 \%$ to $0.375 \%$ ); moreover, external magnetic field could improve relaxation modulus but delay the starting time of relaxation behavior. Secondly, in dynamic experiments, the storage modulus $G^{\prime}$ of MRSPs changed sharply under the stimuli of shear forces with increasing angular frequency, which presented good shear stiffening effect; furthermore, the storage modulus $G^{\prime}$ could be enhanced by stimuli of external magnetic field, which exhibited typical magnetorheological effect; besides, both shear stiffening effect and magnetorheological effect were influenced by temperature, CI volume fraction, and external magnetic field strength. A modified parameter model and a nonperforative particle aggregated chains model which agreed well with experimental data were established, respectively, to describe the shear stiffening and the magnetoinduced behaviors perfectly.

\section{Competing Interests}

The authors declare that there is no conflict of interests regarding the publication of this paper.

\section{Acknowledgments}

This work was supported by Research and Innovation Program of Jiangsu Province for Graduate Students (Grant no. B14042); National Natural Science Foundation of China (Grant no. 51508237); Natural Science Foundation of Jiangsu Province (Grant no. BK20140560); and Research Foundation for Advanced Talents of Jiangsu University (Grant no. 14JDG161).

\section{References}

[1] J. Liang and X.-H. Zhang, "Rheological properties of SP in shock transmission application," Journal of Materials in Civil Engineering, vol. 27, no. 9, Article ID 04014250, 2015. 
[2] R. Cross, "Elastic and viscous properties of Silly Putty," American Journal of Physics, vol. 80, no. 10, pp. 870-875, 2012.

[3] M. P. Goertz, X.-Y.Zhu, and J. E. Houston, “Temperature dependent relaxation of a 'solid-liquid"' Journal of Polymer Science Part B: Polymer Physics, vol. 47, no. 13, pp. 1285-1290, 2009.

[4] S. Wang, S. Xuan, W. Jiang et al., "Rate-dependent and selfhealing conductive shear stiffening nanocomposite: a novel safe-guarding material with force sensitivity," Journal of Materials Chemistry A, vol. 3, no. 39, pp. 19790-19799, 2015.

[5] S. Wang, S. Xuan, Y. Wang et al., "Stretchable polyurethane sponge scaffold strengthened shear stiffening polymer and its enhanced safeguarding performance," ACS Applied Materials \& Interfaces, vol. 8, no. 7, pp. 4946-4954, 2016.

[6] S. Wang, W. Jiang, W. Jiang et al., "Multifunctional polymer composite with excellent shear stiffening performance and magnetorheological effect," Journal of Materials Chemistry C, vol. 2, no. 34, pp. 7133-7140, 2014.

[7] Y. Wang, S. Wang, C. Xu, S. Xuan, W. Jiang, and X. Gong, "Dynamic behavior of magnetically responsive shear-stiffening gel under high strain rate," Composites Science and Technology, vol. 127, pp. 169-176, 2016.

[8] M. R. Jolly, J. D. Carlson, and B. C. Muñoz, "A model of the behaviour of magnetorheological materials," Smart Materials and Structures, vol. 5, no. 5, pp. 607-614, 1996.

[9] Y. Shen, M. F. Golnaraghi, and G. R. Heppler, "Experimental research and modeling of magnetorheological elastomers," Journal of Intelligent Material Systems and Structures, vol. 15, no. 1, pp. 27-35, 2004.

[10] L. Chen, X. L. Gong, and W. H. Li, "Microstructures and viscoelastic properties of anisotropic magnetorheological elastomers," Smart Materials and Structures, vol. 16, no. 6, pp. 26452650, 2007.

[11] F. J. Galindo-Rosales, P. Moldenaers, and J. Vermant, "Assessment of the dispersion quality in polymer nanocomposites by rheological methods," Macromolecular Materials and Engineering, vol. 296, no. 3-4, pp. 331-340, 2011.

[12] Cambridge Polymer Group, “The cambridge polymer group silly putty 'Egg"” 2002, http://www.campoly.com/files/7213/9937/ 8983/CS_CPG_Silly_Putty_Egg_Rheology.pdf.

[13] F. J. Galindo-Rosales, F. J. Rubio-Hernández, and A. Sevilla, "An apparent viscosity function for shear thickening fluids," Journal of Non-Newtonian Fluid Mechanics, vol. 166, no. 5-6, pp. 321325, 2011.

[14] F. J. Galindo-Rosales, F. J. Rubio-Hernández, A. Sevilla, and R. H. Ewoldt, "How Dr. Malcom M. Cross may have tackled the development of 'An apparent viscosity function for shear thickening fluids"' Journal of Non-Newtonian Fluid Mechanics, vol. 166, no. 23-24, pp. 1421-1424, 2011.

[15] T. Tian, G. Peng, W. Li, J. Ding, and M. Nakano, "Experimental and modelling study of the effect of temperature on shear thickening fluids," Korea Australia Rheology Journal, vol. 27, no. 1, pp. 17-24, 2015.

[16] X. Dong, N. Ma, J. Ou, and M. Qi, "Predicating magnetorheological effect of magnetorheological elastomers under normal pressure," Journal of Physics: Conference Series, vol. 412, no. 1, Article ID 012035, pp. 759-773, 2013.

[17] J. C. M. Garnett, "Colours in metal glasses and in metallic films," Philosophical Transactions of the Royal Society A: Mathematical, Physical and Engineering Sciences, vol. 203, pp. 385-420, 1904.

[18] K. K. Kärkkäinen, A. H. Sihvola, and K. I. Nikoskinen, "Effective permittivity of mixtures: numerical validation by the FDTD method," IEEE Transactions on Geoscience \& Remote Sensing, vol. 38, no. 3, pp. 1303-1308, 2000.

[19] O. Wierner, Die Theorie des Mischkörpers für das Feld der stationären Strömung. Erste Abhandlung: Die Mittelwertsätze für Kraft, Polarisation und Energie, 1912.

[20] Y. M. Shkel and D. J. Klingenberg, "Magnetorheology and magnetostriction of isolated chains of nonlinear magnetizable spheres," Journal of Rheology, vol. 45, no. 2, pp. 351-368, 2001.

[21] A. Dorfmann and R. W. Ogden, "Magnetoelastic modelling of elastomers," European Journal of Mechanics. A. Solids, vol. 22, no. 4, pp. 497-507, 2003. 

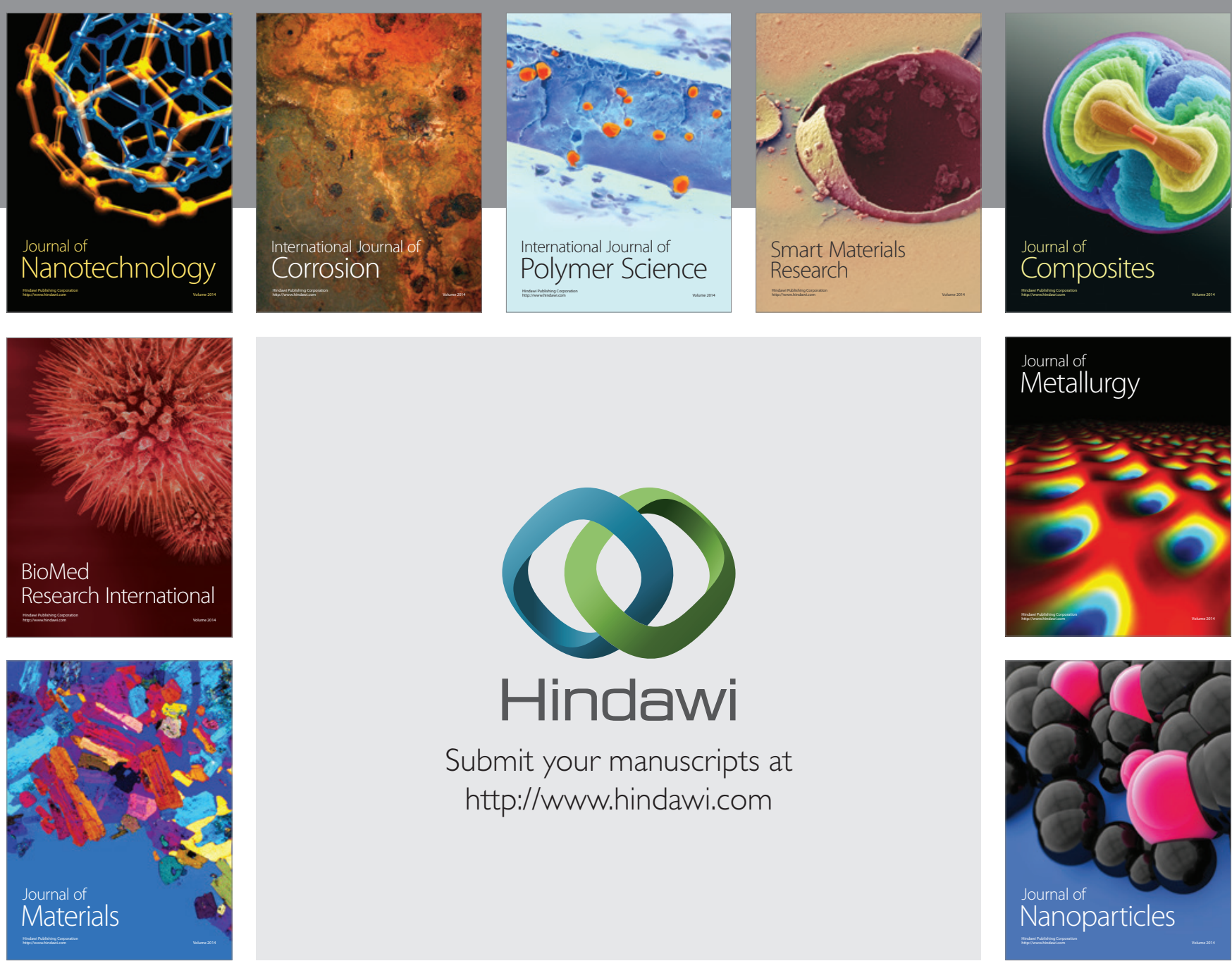

\section{Hindawi}

Submit your manuscripts at

http://www.hindawi.com

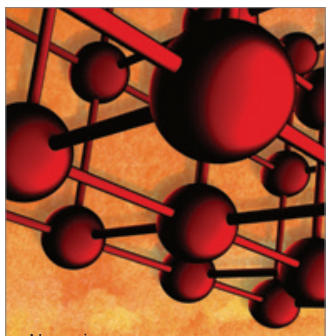

Materials Science and Engineering
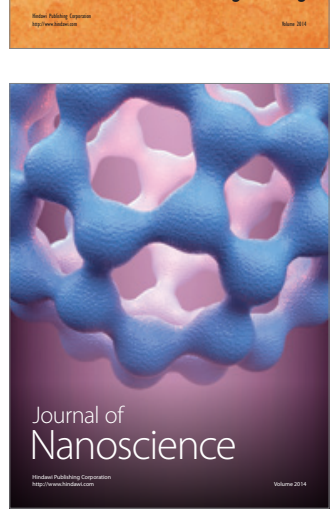
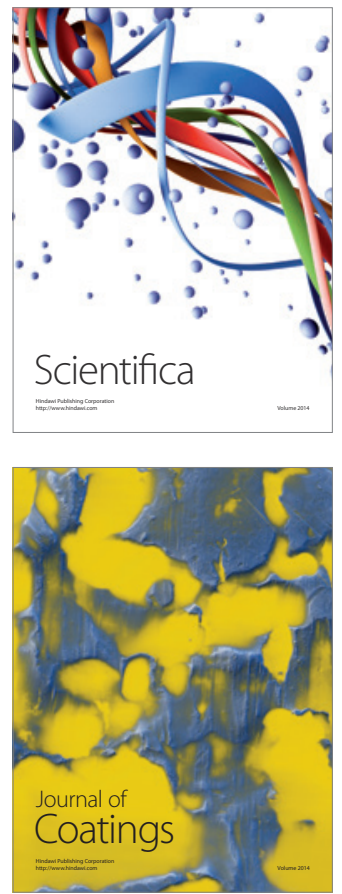
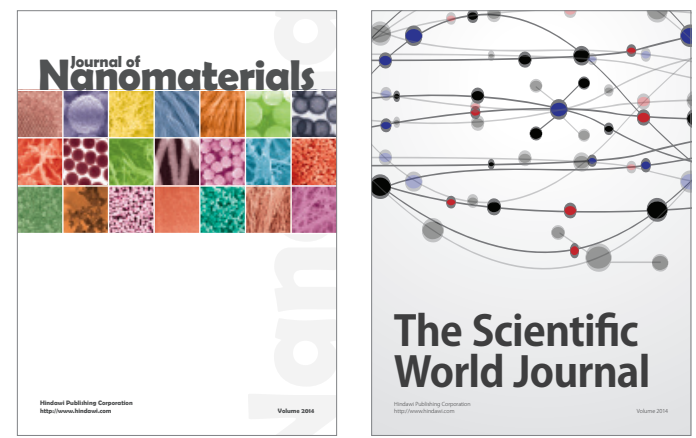

The Scientific World Journal
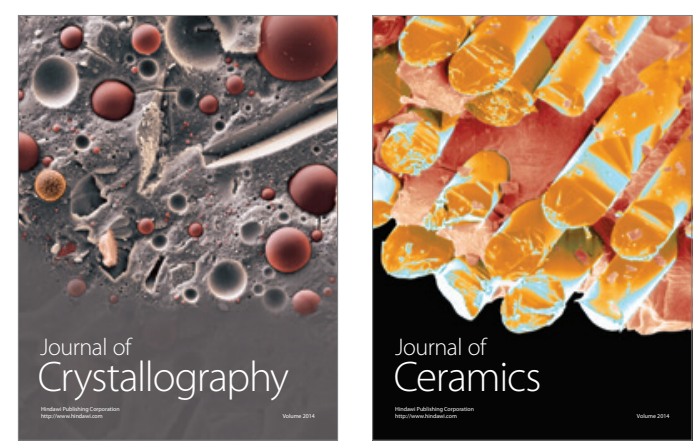
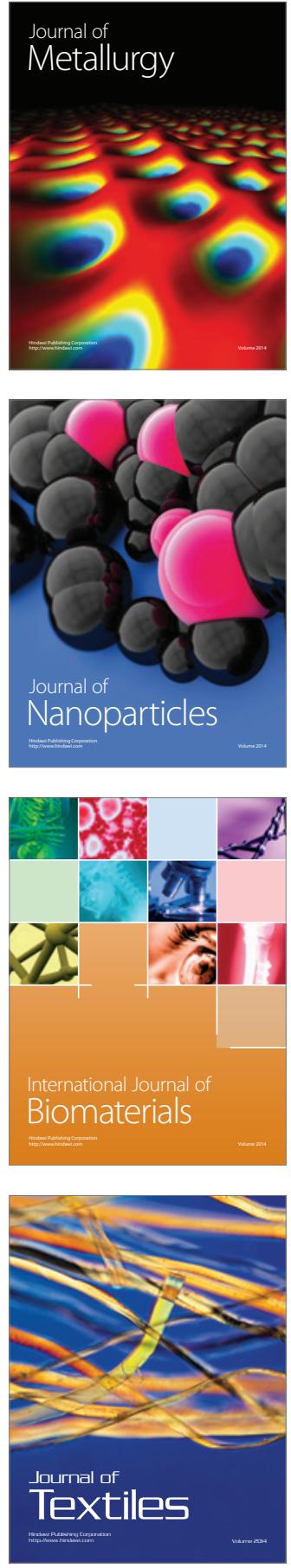